\title{
LPG GAS LEAKAGE DETECTION USING
}

\section{IOT}

\author{
Dr. Chetana Tukkoji \\ Assistant Professor, Dept. of CSE \\ GITAM School of Technology, Bengaluru
}

\begin{abstract}
This paper provides a brand new approach to discover LPG discharge supported microcontroller based Arduino. To alert on Liquefied rock oil Gas (LPG) leakage and preventing any unwanted incident, we need to apply some cautions to discover the discharge. It can be developed associate degree Arduino based LPG gas detector alarm, if gas leakage happens. The LPG detector MQ6 is associate degree correct LPG sensing device that acquires the signal intensity. Associate degree economical Arduino based signal process mechanism is followed that effectively quantizes the non-inheritable electrical signal. The intensity of the LPG leakage is classed into 3 categories, such as LOW, MEDIUM and HIGH based on square measure. This paper conjointly shows the ratio and temperature over the alphanumeric display. The importance and connection of the paper is very beneficiary for man as a result of it's a vital cautions for our domestic life.
\end{abstract}

Keyword: IOT, Ardunio, LPG, Sensors

\section{INTRODUCTION}

LPG is that the abbreviation or short kind for liquefied oil gas. Like all fossil fuels, it's a non-renewable supply of energy. It is extracted from fossil oil and gas. The most compositions of LPG square measure Hydrocarbons containing three or four carbon atoms. The conventional parts of LPG so, square measure gas (ClHa) and alkane (CaHro). Tiny concentrations of alternative hydrocarbons may additionally be gift betting on the supply of the LPG and the way it's been created, parts apart from hydrocarbons may additionally be gift. LPG is extremely combustible and should thus be hold on off from sources of ignition and during a well-ventilated space, in order that any run will disperse safely. LPG vapors is heavier than air thus care ought to be taken throughout storage in order that any run won't sink to the bottom and find accumulated in a district that is low lying and tough to disperse. LPG gas is largely gas and alkane and it's

\author{
Mr. Sanjeev Kumar A. N \\ Assistant Professor, Dept. of CSE \\ GITAM School of Technology, Bengaluru
}

scentless in its state of nature. The smell that we tend to notice once there's a run is really of a wholly totally different agent, referred to as alkyl radical Mercaptan. This substance is additional to the gas once it leaves the most storage terminals [1].

The prime aim of paper is to detect Gas leakage in home, hotels, schools and other domestic areas, and gives alert message to the surrounding people. Nowadays Gas sensors are being used globally in the field like safety, health, instrumentation etc. This paper is an implementation of the same using MQ-5 gas sensor and DHT11 temperature sensor. The MQ5 sensor is commonly used for detecting gas leakage for various applications and the DHTIL is used for measuring the humidity and temperature of surrounding area. The device also keeps displaying the leakage amount and humidity \& temperature on an LCD display. The MQ6 gas sensor detects the concentration of gas in ppm and outputs analog value which can be converted to a digital signal using inbuilt Analog to Digital Convertor of Arduino. The paper allows the user to set the low, medium and dangerous level for leakage based on the same digital measure. The intensity values are compared with two predetermined thresholds and based on that, it classifies it into three different classes of concentration of leakage [2].

\section{LITERATURE SURVEY}

Abhijeethrathi et.al (2013); introduced a golem based on automatic gas detection and indication golem. They planned image depicts a mini mobile golem that is capable to observe gas leak in unsafe places. Whenever there's an occasion of gas leak during a specific place the golem instantly scan and sends the information to golem mobile through wireless communication like Bluetooth. We have a tendency to develop a golem application for golem primarily based good phones which may receive knowledge from golem directly through Bluetooth. The applying warns with a sign whenever there's an occasion of gas leak and that we can even management the golem 
movements via Bluetooth by exploitation text commands yet as voice commands. The previous mobile robots a supported heterogeneous technologies like GSM, GPS, net primarily based etc., however the most disadvantage of these prototypes were the absence of communication specially areas. So, with the speedy developments and tremendous changes in technology we've ample techniques to eradicate previous issues. Wireless communication protocols play an important role in gift trends. Bluetooth, Wi-Fi, Zigbee etc., we have a tendency to use one among the simplest feature of good phone, i.e., the Bluetooth technology to regulate and monitor parameters driven by a golem [3].

In the slide share document (2014); They introduce a golem and mobile application for the in the meantime, the system image has impressively incontestable its use and capability in intensive series of tests. The drive unit, the navigation system and, therefore, the complementary detector systems performed fantastically throughout the tests. Moreover, it helps to avoid practice of human inspectors in in all probability dangerous environments. However, before activity in industrial settings, a lot of development is required (e.g., in explosion protection, package development, etc.), and if truth be told legal issues ought to be processed before activity in business settings. Still, it's sure that AN autonomous, mobile gas detection and leak localization golem is feasible these days and might considerably enhance safety [4].

Pal-Stefan et.al (2008); Introduced few old and new technologies to detect the gas. In this the proposed techniques are nontechnical, acoustic methods, optical methods and active methods. Survey says wide range of techniques are available for Gas detection. However, each applications has few of the limitations [5]

Manichandana Simrah et.al (2019); in this paper they told about their research on leakage detection and analysis of leakage point in the gas pipeline system. In this paper they gave various model which used SCADA I/F Model: The SCADA system has the function of transferring the acquired data from a pipeline system to Transient Simulation Model every 30 seconds. This module communicates with SCADA. Dynamic parameters are collected every 30 seconds, such as pressure, flow and temperature. Transient Simulation Model: Transient flow is simulated utilizing perfect numerical methods based on actual data. Pressure and temperature served as independent variables are provided in order to get average pressure and average temperature [6]
Rahul Nalawade et.al, (2018); in this paper ARM7 primarily based machine-driven high performance system is used for LPG refill booking and outpouring detection. That decreases the outpouring resistance. Microcontroller sends a message "EMERGENCY ALERT: LPG gas outpouring found in your home to needed cell numbers via GSM module and therefore the same are going to be displayed on digital display. This technique detects the outpouring of the LPG associated alerts the patron regarding the leak by SMS and as an emergency live the system can shut down the ability offer, whereas activating the alarm [7].

Vasudev Yadav et.al (2016); in this paper MQ-9 computer circuit is used associated with embedded system. Such systems sometimes haven't any keyboard, screen, disks, printers, or different recognizable I/O devices of a private $\mathrm{pc}$, and will lack human interaction device. MQ-9 sensor that activate a buzzer and alert the semiconductor diode within the event of gas [8].

\section{SYSTEM ARCHITECTURE}

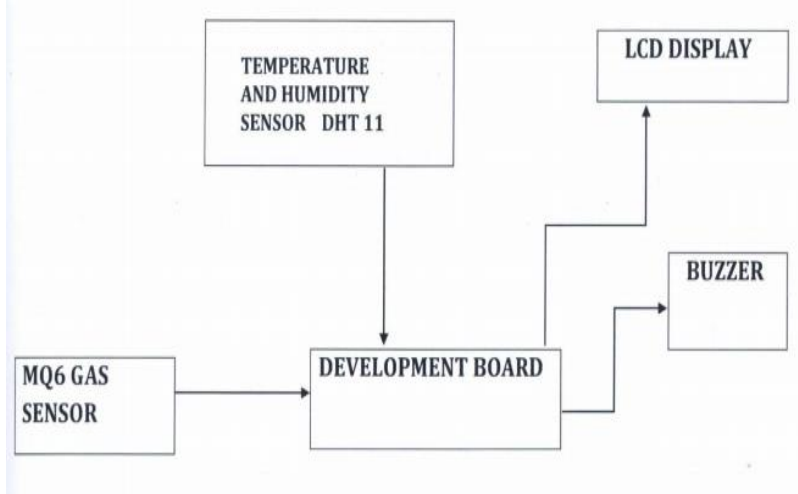

Figure-1. System Architecture

In the scribd document (2013) mentioned how LPG Gas is important, While LPG is an important would like of each social unit, its leak may lead to a disaster. To alert on LPG leak and forestall any miss happening there square measure varied product to observe the leak. Here we have developed associate degree Arduino based LPG gas detector alarm. If gas leak happens, this technique detects it associate degreed makes an alert by a buzz the buzzer hooked up with the circuit. This technique is straightforward to create and anyone World Health Organization have some data of physics and programming to build [9]. 


\author{
1. Arduino Pro Mini \\ 2. LPG Gas sensor Module \\ 3. Buzzer \\ 4. BC 547 Transistor \\ 5. $16 \times 2 \mathrm{LCD}$ \\ 6. $1 \mathrm{~K}$ resistor \\ 7. Bread board \\ 8. 9 volts battery \\ 9. Connecting wires
}

Components of System Architecture

\section{ARDUINO UNO}

Arduino Uno board provides the engineers, artist, designers, amateur and anyone WHO tinker with technology with a lost-cost, straightforward to use technology to form inventive, interactive object, and platform supported Arduino boards that plugged into Associate in Nursing output - activating a motor, turning on Associate in Nursing semiconductor diode, business enterprise one thing online, we will be training our board what to try to by causing a collection of directions to the microcontroller on the board thus we employ the Arduino artificial language (based on wiring), and also the Arduino software system (IDE) supported process. The Arduino software system is easy-to-use for beginners, however versatile enough for advanced users.

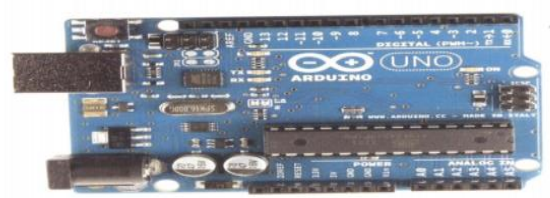

Figure-2: Arduino software system

\section{MQ6 GAS SENSOR}

In the Youtube, experts have explained about different components of LPG architecture. The sensing element is capable of detecting6* differing kinds of combustible gases on label sensitivity. This sensing element may be label mistreatment the potentiometer fitted within the jailbreak board of MQ6 gas sensing element. The sensing element provides associate degree analog output. The MQ-6 will observe gas concentrations in any place from two hundred to $10000 \mathrm{ppm}$. The sensor's output is associate degree analog resistance. Interfacing with the sensing element module is finished through a 4-pin board compatible Slp header and needs one i/o pin from the host microcontroller. The aboard microcontroller give initial heating interval once power-up and so starts to live LPG sensing element output. Low frequency it found the LPG concentration higher than predetermined worth, it'll inform the Host controller by pull the output pin to High and Starts to blink aboard diode. The sensing element module is especially supposed to produce a method of scrutiny LPG supply's associate degreed having the ability to line an alarm limit once the source becomes excessive [10].

It consists of four pins, the details of LPG Gas sensor is shown in Table-1.

\begin{tabular}{|l|l|l|l|}
\hline Pin No & Pin Name & I/0 & \multicolumn{1}{|c|}{ Details } \\
\hline 1 & VCC & Power IN & Positive power supply, 5v Regulated Power \\
\hline 2 & GND & Power GND & Ground \\
\hline 3 & ANALOG & $0 /$ P & Analog output voltage \\
\hline 4 & OUT & $0 / P$ & Output voltage \\
\hline
\end{tabular}

Table-1. Pin Specification

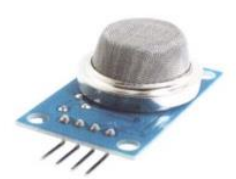

Table-2. 16x2 LCD DISPLAY

Table-2 it is Digital Display, where it uses liquid to supply a noticeable image, and every character are fabricated from $5 \times 8$ picture element dots.

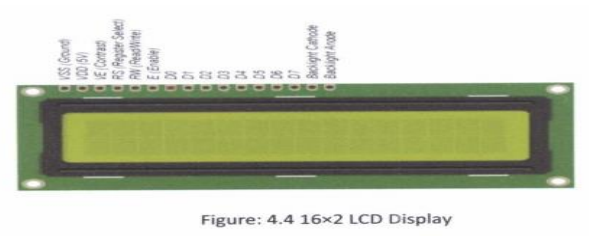

Figure-3. Features of LCD module

Operating Voltage is $4.7 \mathrm{~V}$ to $5.3 \mathrm{~V}$. Current consumption is $1 \mathrm{~mA}$ without backlight. Where it displays alphabets and numbers. It consists of two rows, where it prints 15 characters in each row, with $5 \times 8$ pixel box. Characters are printed in Green and Blue Backlight.

\section{CONNECTING WIRES}

A wire may be a single, typically cylindrical, versatile strand or rod of metal. Wires square measure accustomed bear mechanical masses or electricity and telecommunications signals. Wire is often fashioned by drawing the metal through a hole in a very die or draw plate. Wire gauges are available in numerous customary sizes, as expressed in terms of a gauge range. The term wire is additionally used additional 
loosely to confer with a bundle of such strands, as in "multi stranded wire", that is additional properly termed a wire rope in mechanics, or a cable in electricity. Connecting wires will be made up of $\mathrm{Al}$ and different materials the majority electrical wires square measure fabricated from copper.

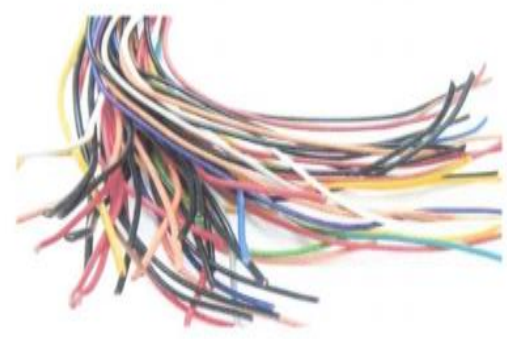

Figure-4. To depict the connecting wires

\section{JUMPER WIRES}

Akarsh Agarwal et.al (2019, 2018), A jumper wire is associate electrical wire, or cluster of them during a cable, with a connecter or pin at every finish (or typically while not them - merely "tinned"), that is often accustomed interconnect the parts of a board or different model or take a look at circuit, internally or with different instrumentation or parts, while not fastening. Individual jump wires ar fitted by inserting their "end connectors" into the slots provided during a board, the header connecter of a printed circuit, or a chunk of equipment $[11,12]$.

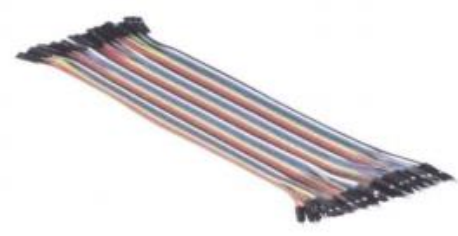

Figure-5. Jumper Wires

\section{BUZZER (Piezo Electric)}

A buzzer or pager is AN audio signaling device, which can be mechanical, mechanical device, or electricity (Piezo for short). It has 2 pins in it. It is easy construction and low worth create it usable in varied applications like car/truck reversing indicator, computers, decision bells etc. [13]. It is that the phenomena of generating electricity once mechanical pressure is applied to sure materials and also the other way around is additionally true. Piezo electrical materials square measure either naturally out there or manmade. Piezo ceramic is category of manmade material, that poses Piezo electrical impact and is wide accustomed create disc, the center of Piezo buzzer. Once subjected to AN alternating field of force they stretch or compress, in accordance with the frequency of the signal thereby manufacturing sound.

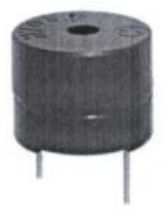

Figure-6. Buzzer

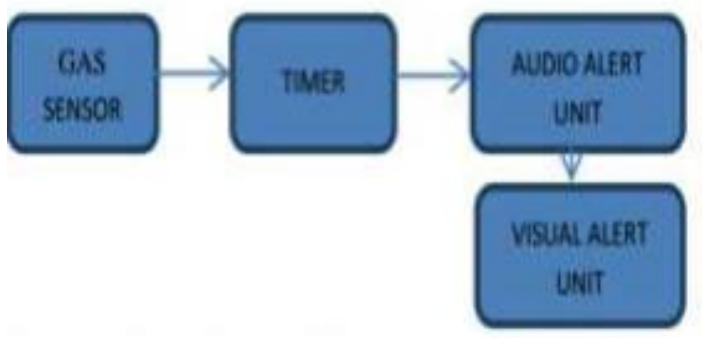

Figure 7: Block Diagram of the User Interactive Gas Leakage and Fire Alarm System

The device that was planned during this paper is MQ nine. It advantage is that it has; sensible sensitivity to $\mathrm{CO} / \mathrm{Combustible} \mathrm{gas,} \mathrm{high} \mathrm{sensitivity} \mathrm{to} \mathrm{gas,} \mathrm{fuel} \mathrm{and}$ $\mathrm{CO}$, long life and low price, easy drive circuit. The enclosed MQ-9 has half-dozen pins, four of that square measure accustomed fetch signals, and alternative two for providing heating current. The device works with voltages between $5 \mathrm{~V}$ and $12 \mathrm{~V}$ AC or DC. A $5 \mathrm{~V}$ offer voltage was used for this style [14].

\section{PROPOSED SYSTEM}

Here we have adapted new technology IOT (Internet of Things) to get fastest notification of gas leakage. We shall use a stepper motor to OFF the knob of cylinder regulator to avoid the accidental cases due to gas leakage. We will also use a website or application under the IOT technology to get fastest response from the module. The other module and things which are used in this paper is GSM module, microcontroller, exhaust fan, LED for indication, a buzzer to notify local peoples. And MQ 5 or MQ 6 gas sensor module to sense the gas leakage.

In this proposed model we want to achieve few aspects: To Design an Embedded System we need, modules refer: Table-3 


\begin{tabular}{|l|l|}
\hline Modules & Purpose \\
\hline AVR microcontroller & To control all the module and things \\
\hline Accident Avoiding Feature & Used exhaust fan to reduce the gas from the place \\
\hline Stepper motor & To OFF the cylinder knob \\
\hline Buzzer & To notify the local people automatically by weeping buzzer \\
\hline IOT Module & For app notification \\
\hline Sensors & MQ 5 or MQ 6 - To sense the gas leakage \\
\hline
\end{tabular}

Table-3: System Modules and its uses

The main advantage of this paper is that it can determine the leakage and send the data over to a website, where it can be monitored and corrective actions can be taken. If appropriate measures are taken quickly after it is reported over the IOT, it can help in saving the loss of lives and property.

\section{SYSTEM ANALYSIS AND DESIGN}

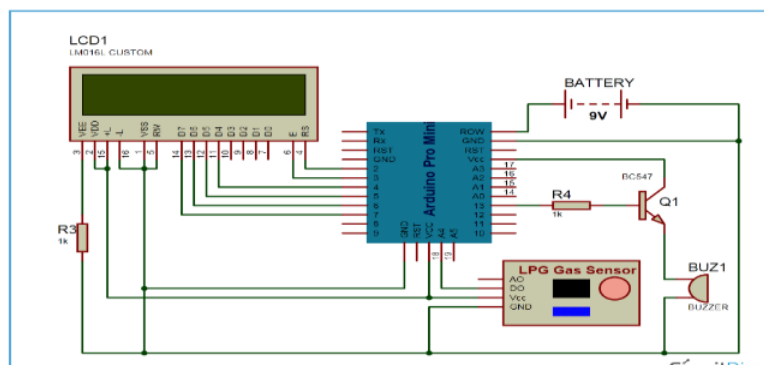

Figure-8: System Design

While LPG is a vital would like of each house, its outpouring could lead on to a disaster. To alert on LPG outpouring and forestall any miss happening there area unit numerous product to notice the outpouring. Here we've got developed associate Arduino primarily based LPG gas detector alarm. If gas outpouring happens, this technique detects it associated makes an alert by droning the buzzer connected with the circuit. This technique is simple to create and anyone WHO have some information of physics and programming, will build it.

As shown within the schematic diagram higher than, it contains Arduino board, LPG GAS device Module, buzzer and 16x2 liquid crystal display module. Arduino controls the complete method of this technique like reading LPG Gas device module output, causation message to liquid crystal display and activating buzzer. We are able to set sensitivity of this device module by intrinsic potentiometer placed on that.

The functioning of the circuit once the device is supercharged ON. Initial the microcontroller initializes the show LCD digital display alphanumeric display and starts reading the analog voltage from the MQ-6 device. The MQ5 device gas module has four pins. 2 pins area unit used for interfacing with development board and different 2 pins area unit VCC and ground. Out of 2 interfacing pins one pin is analog output and different is digital pin. The analog output pin of the module is employed for detective work concentration level of gas outpouring and interfaced with the $\mathrm{A} 0$ analog input pin of the Arduino board. The analog voltage from the device is digitized victimization the in-built $\mathrm{ADC}$ channel and keep in a very variable as a 10 -bit worth. The $16 \mathrm{X} 2$ show $\mathrm{LCD}$ digital show| alphanumeric display display is employed to display the worth of gas concentration. It is connected to the Arduino board by connecting its knowledge pins to pins 4 to 7 of the Arduino board. The $\mathrm{RS}$ and $\mathrm{E}$ pins of the liquid crystal display area unit connected to D2 and D3 pins of the Arduino UNO severally. The RW pin of the liquid crystal display is connected to the bottom. The device worth is compared with a label threshold and if the device worth exceeds that worth, the buzzer gets activated. The buzzer is connected to the weight unit pin of the Arduino. A $10 \mathrm{k}$ potentiometer is additionally connected to the liquid crystal display pin contagion, the battery and therefore the ground. This can be used for the distinction on the show LCD digital display alphanumeric display of the written alphabets or digits [15].

\section{RESULTS AND DISCUSSIONS}

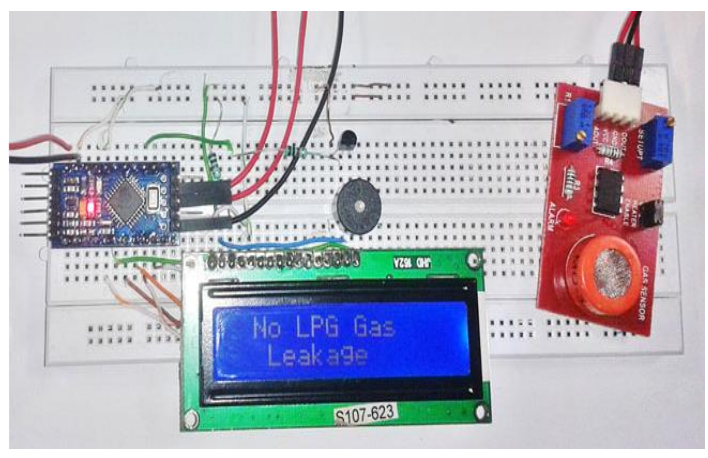

Figure-9: System Display Unit

Safety plays a serious role in today's world and it's necessary that smart safety systems are to be enforced in places of education and work. The LPG or gas that is combustible mixture of organic compound gases utilized in use as fuel in abundant application like homes, hostels, industries, automobiles' vehicles attributable to its fascinating properties that embrace high hot price, that manufacture the less smoke, 
produces less soot and doesn't cause abundant hurt to the setting. Each cases burns to provide clean energy, but there's a significant drawback concerning their outpouring within the air. The gases being heavier than air don't disperse simply could and should and will cause suffocation once indrawn conjointly once gas outpouring into the air may cause explosion' thanks to the explosion of LPG gas the no of deaths has been inflated in recent years. Thus this device are often used to avoid these issues by sleuthing and conjointly preventing outpouring of LPG. Gas leak discovering is that the method of characteristic doubtless venturesome gas leaks by means that of varied sensors 'The advantage of this automated detection And alerting system over the manual technique is that it offers fast latency And correct detection of an emergency and successively leading quicker diffusion of the vital situation' $r$ The gas detection an alter system that we've designed could be a terribly value effective system to detect outpouring of any gas. It is not solely detects outpouring of gas it conjointly alerts U.S. by manufacturing appears like alarm etc. It has its applications in numerous fields like faculty, colleges, universities, homes and industries.

\section{ACKNOWLEDGEMENT}

I would like to thank my brother for helping me out to complete this paper. I also thank our students for supporting me for giving information about related topics.

\section{CONCLUSION AND FUTURE ENHANCEMENT}

Gas escape could result in severe accidents which ends in material losses and human injuries. Gas escape happens chiefly because of poor maintenance of apparatus and inadequate awareness of the individuals. Thus LPG escape detection are useful to stop accidents and to avoid wasting human lives. This paper conferred LPG escape detection and alert system. This technique triggers buzzer and displays the severity of the escape to alert individuals once LPG escape is detected. This technique is incredibly straightforward nevertheless reliable.

It leaves United States with the additional scope of improvement. Battery utilized in this technique is of $5 \mathrm{~V}$ that isn't that a lot of tolerable, in future improvement, we are able to use a much bigger, reversible one, which may sustain the gas detection module for an extended amount of your time, with alert whenever battery runs out' In additional modification, additionally to solely escape detection we are able to resolve the concentration of the gas too. The paper includes a smart viability to be launched in industrial market, tiny scale industries having multiple cylinders hold on. With additional improvement in style the system will be created additional handy and price effective for the users.

\section{REFERENCES}

1.Vaishnavi et.al (2014) "Intelligent LPG Leakage Detection", International Journal Of Scientific \& Engineering Research, Vol. 5, Issue 11, 2014.

2.http://Centrallibrary.Cit.Ac.In/Dir/Project\%20Repo rt/2018/Diploma/ETE/Detection\%20of\%20lpg\%20le akage\%20using\%20arduino.Pdf

3.https://Www.Slideshare.Net/Abhijeetrathi/Lpg-

Detection-Mechatronic-System

4.https://Www.Scribd.Com/Document/436529826/D etection-Of-Lpg-Leakage-Using-Arduino-Pdf

5.http://Www.Aut.Upt.Ro/ Palstefan.Murvay/Papers/ Survey_Gas_Leak_Detection_Localization_Techniqu es.

6. Manichandana,Simrah et.al (2019), “Survey Paper On Gas Leak Detection Using Iot" JASC: Journal Of Applied Science And Computations Volume VI, Issue I, January/2019 ISSN NO: 1076-5131, Page No: 65100.

7. Rahul Nalawade et.al (2018) "Iot Based Gas Leakage Detection and Alert Generation",

International Journal of Future Revolution in computer science \& communication engineering ISSN: 2454-4248, 2018, volume-4, Issue-5, 175-176. 8.Vasudev Yadav, et.al. (2016), "A Review On Microcontroller Based Lpg Gas Leakage Detection \& Controlling System Using Iot \& Gsm Module", IEEE 2nd International Conference On Electronics Technology (ICET), Published 2016, Corpus ID: 209328541.

9.https://Www.Scribd.Com/Document/404213286/M icrocontroller-Based-LPG-Leakage-Detect-1-Docx. 10.https://Www.Youtube.Com/Watch?V=877wiaanit 11. Akarsh Agarwal et.al (2019), "R.F.L. Safety Kit for Domestic LPG", International Journal of Innovative Research in Electrical, Electronics, Instrumentation and Control Engineering, IJIREEICE, Vol. 7, Issue 5, May 2019. DOI 10.17148/IJIREEICE.2019.7514. 
12.http://www.aut.upt.ro/ palstefan.murvay/papers/s urvey_gas_leak_detection_localization_techniques.p df

13.https://www.flir.com/discover/instruments/gasdetection/remote-detection-and-localization-of-gasleaks-with-autonomous-mobile inspection-robots-intechnical-facilities/

14.https://docplayer.net/153343597-A-survey-paperon-gas-leak-detection-using-iot.html.

15.https://wenku.baidu.com/view/f8dc5e32be23482f

b4da4c69.html 